\title{
Xerostomia after Radiotherapy for Oral and Oropharyngeal Cancer: Increasing Salivary Flow with Tasteless Sugar-free Chewing Gum
}

\author{
Julie Killerup Kaae, Lone Stenfeldt and Jesper Grau Eriksen* \\ Department of Oncology, Odense University Hospital, Odense, Denmark
}

Introduction: Radiation-induced xerostomia is a frequent late side effect after treatment for oral and oropharyngeal cancers. This may induce swallowing difficulties, compromised oral well-being, reduced nutrition intake, or speech deficiencies. Consequently, quality of life is often impaired for these patients.

Objectives: The purpose of this study was to investigate the possibility to mechanically stimulate residual saliva function by using tasteless and sugar-free chewing gum. It was hypothesized that tasteless and sugar-free chewing gum could immediately increase salivary flow and potentially improve oral well-being when used on a regular basis.

Methods: From October to December 2014, 31 consecutive patients treated with primary

OPEN ACCESS

Edited by:

Amanda Psyrri,

Attikon University Hospital,

Greece

Reviewed by:

Richard Simcock,

NHS, UK

Ourania Nicolatou-Galitis, University of Athens, Greece

*Correspondence:

Jesper Grau Eriksen

jesper.grau.eriksen@rsyd.dk

Specialty section: This article was submitted to Head and Neck Cancer,

a section of the journal

Frontiers in Oncology

Received: 06 November 2015 Accepted: 18 April 2016

Published: 03 May 2016

Citation:

Kaae JK, Stenfeldt $L$ and Eriksen JG (2016) Xerostomia after Radiotherapy

for Oral and Oropharyngeal Cancer: Increasing Salivary Flow with Tasteless Sugar-free Chewing Gum.

Front. Oncol. 6:111.

doi: 10.3389/fonc.2016.00111 radiotherapy $(\mathrm{RT})$ and concomitant cisplatin (in locally advanced cases) for oral or oropharyngeal cancer consented to participate. All patients had finalized RT 2-8 months prior to participation and suffered from xerostomia. Samples of unstimulated and chewing gum-stimulated saliva were obtained at the entry into the study (Visit 1). For 2 weeks, patients used chewing gum on a regular basis whereupon saliva measurements were repeated to verify the changes (Visit 2). An abbreviated EORTC H\&N35 questionnaire was completed for both visits. A small control group consisting of young and healthy individuals also tested the chewing gum.

Results: Twenty patients completed the study and an increase in saliva flow was observed for 14 patients. Before and after intervention with chewing gum, an increase in mean saliva output was seen between unstimulated and stimulated saliva for both Visit 1 and 2 ( $p=0.008$ and $p=0.05$, respectively). No change in saliva output was seen in the control group.

Conclusion: The chewing gum was able to stimulate saliva output that was seen at the beginning and at the end of the intervention. No improvement in baseline saliva was seen. Relevant changes in subjective measures of xerostomia were seen after 2 weeks of chewing the gum.

Keywords: xerostomia, chewing gum, radiotherapy, oral cancer, oropharyngeal cancer

\section{INTRODUCTION}

Xerostomia is a common acute and late side effect when treating head and neck cancer (HNC) patients with curative intended radiotherapy (RT) (1-3). Xerostomia is the subjective feeling of oral dryness, whereas hyposalivation is the physiological reduction in salivary flow (4). Hyposalivation is defined as unstimulated whole saliva flow of $\leq 0.2 \mathrm{~mL} / \mathrm{min}$, and symptoms of xerostomia often 
become evident when saliva flow is below $0.1-0.2 \mathrm{~mL} / \mathrm{min}(5,6)$. Xerostomia is not necessarily correlated to a reduction in saliva flow and may also be present independent of hyposalivation. Healthy individuals produce between 0.5 and $1.5 \mathrm{~L}$ of saliva daily, with saliva being secreted by the three paired major salivary glands. It consists of approximately $99 \%$ water and $1 \%$ proteins and salt, and normal daily secretion of saliva is vital for maintaining good oral health, nutritional intake, and communication skills $(7,8)$.

The severity of xerostomia caused by RT is affected by the total dose and dose per fraction and is often irreversible $(9,10)$. To assess the severity of xerostomia in the clinic, a good approach is to make use of an observer-based scoring system, and a validated quality of life measurement device and measuring salivary flow (11). Various treatments are available to relieve the discomfort of xerostomia, including symptomatic relief by oral lubricants and saliva substitutes. Salivary stimulants may be considered where residual salivary gland function remains (12).

The purpose of this study was to investigate the possibility to mechanically stimulate residual saliva function. It was hypothesized that by using a tasteless and sugar-free chewing gum, an immediate increase in saliva flow would be obtained and potentially improve oral well-being when used on a regular basis.

\section{MATERIALS AND METHODS}

This non-randomized cohort study was conducted at the Department of Oncology at Odense University Hospital from October to December 2014. HNC patients diagnosed with oral or oropharyngeal carcinomas were eligible for participation after completing either curative intended treatment with intensitymodulated radiation therapy (IMRT) or postoperative IMRT with or without concomitant cisplatin.

Patients were recruited during follow-up visits to the department 2-12 months after having completed treatment. Furthermore, all patients had to suffer from xerostomia (slight/ moderate/severe) as graded by the attending physician according to the Danish Head and Neck cancer Group (DAHANCA) follow-up guidelines. Medical records for all consenting patients were reviewed with regard to medication-induced xerostomia.

For each patient, the study consisted of two visits at the department separated by 14 days. All oral intakes $1 \mathrm{~h}$ before onset of the test, which potentially could influence the test results, were not recommended. Visit 1 consisted of four elements:

(1) An unstimulated saliva sample.

(2) An abbreviated version of the EORTC H\&N35 questionnaire.

(3) Testing the chewing gum.

(4) A stimulated saliva sample.

First, the unstimulated saliva sample was obtained by the patient spitting into a test tube for $5 \mathrm{~min}$. No sipping of water or saliva stimulant was allowed $1 \mathrm{~h}$ before and during the collection of the saliva sample. Second, an abbreviated version of EORTC H\&N35 questionnaire (including only the oral and food-related questions plus two additional questions added for the purpose of the study) (Table 2) was completed to asses xerostomia grade, quality of life, and difficulties associated with xerostomia $(13,14)$. Afterward, a saliva stimulant in the form of a tasteless sugar-free chewing gum was tested and chewed for 5 min supported by a metronome ( 60 beats/min). After depositing the chewing gum, the stimulated saliva sample was obtained by the patient spitting into a second test tube for $5 \mathrm{~min}$.

The study chewing gum was distributed for use at home inbetween visits, and patients were instructed to use it three to five times a day including before regular meals.

At Visit 2, the patient repeated all tests conducted during Visit 1. A second unstimulated and stimulated saliva sample was obtained, and the EORTC H\&N35-abbreviated questionnaire was completed again.

\section{Chewing Gum}

For this study, a specially designed chewing gum to stimulate whole saliva flow was used. The chewing gum contained neither taste nor sugar, had no hard coating, and consisted of a basic gum base added mannitol. The chewing gum conformed to the relevant European Union legislations on foods. For practical use, it was reduced in size to accommodate any inconvenience for the recruited patients dealing with pain in the mouth or having trouble overcoming large food objects.

\section{Control Group}

To compare and evaluate the saliva stimulating effects of the chewing gum, the product was tested on a small control group ( $n=10$ ) consisting of young, healthy, non-smoking, and nonmedicated students/health-care professionals. None reported problems regarding xerostomia. All participants were instructed in the spitting and chewing procedures and provided saliva samples. One unstimulated and stimulated saliva sample was obtained from each participant.

\section{Saliva Measurement}

Saliva samples were weighted and the salivary flow rate was calculated in grams per minute (7). For all sample tubes pre- and postweight were measured on a Mettler Toledo (Colombus, $\mathrm{OH}$, USA) weight. To determine the exact weight of saliva, the weight was calibrated with 500,100, and $1 \mathrm{~g}$ weights before use. After weighting, all samples were centrifuged at $2000 \times g$ and $20^{\circ} \mathrm{C}$ for $5 \mathrm{~min}$ before the volume was determined by use of $3 \mathrm{~mL}$ pipettes. For this study, the weight of the saliva output was considered most accurate.

\section{Ethics}

Written informed consent was obtained from all patients agreeing to participate. The study was approved by the Regional Scientific Ethical Committes for Southern Denmark. The study was conducted and the data analyzed without involvement from Fertin Pharma who produced the chewing gum.

\section{Statistics}

The primary endpoint was the immediate increase in saliva output after stimulation with tasteless sugar-free chewing gum at Visit 1. 
The secondary endpoints included oral well-being estimated by the abbreviated EORTC H\&N35 questionnaire.

Saliva output was expected to be normally distributed. The correlations between measurements of the saliva output were tested using a paired $t$-test. Patient and tumor characteristics for all eligible patients and responses to the questionnaires were tested by Spearman's correlation for categorical values. A two-sided $p$-value $<0.05$ was regarded as significant. Data were analyzed using SPSS version 22 for Windows.

\section{RESULTS}

A total of 62 consecutive HNC patients were assessed for eligibility. Thirty-one patients declined participation. Among the 31 included patients, 11 patients did not complete due to problems with prosthetic teeth $(n=2)$, distance to the hospital $(n=2)$, withdrawal of consent without explanation $(n=5)$, or screening failure $(n=2)$. In total, 20 patients completed the study and underwent saliva collection 4 times while testing the chewing gum during the 2-week study period.

The study group consisted of 15 men and 5 women, age ranging from 46 to 73 years (median 62 years) and all current non-smokers. Considering the follow-up period after RT, 10 patients had completed treatment within $2-5$ months, whereas 10 patients had finished within 6-12 months. The patients declining participation did not differ from the group of patients consenting to participation with regard to gender, age, follow-up after RT, tumor site, clinical stage, or distance to hospital. Only smoking tended to be more prominent among patients who did not enter the study $(p=0.01)$ (Table 1$)$.

\section{Xerostomia}

All study patients reported xerostomia before testing the chewing gum. Responses to the abbreviated EORTC H\&N35 questionnaire at Visit 1 showed that xerostomia was a major complaint, with $90 \%$ of the patients rating xerostomia as "quite a bit" or "a lot" (Table 2). At Visit 2, only $30 \%$ of the patients rated xerostomia as "quite a bit," and none experienced complaints corresponding to "a lot." No significant difference was found for the patientreported evaluations of xerostomia. Medical records revealed that five patients were prescribed opioids, antidepressants, and antiepileptic medication, and all five patients had pronounced xerostomia complaints ("quite a bite" or "a lot").

\section{Oral Complaints}

Pain in the mouth or jaw and eating difficulties were also evaluated with the abbreviated EORTC H\&N35 questionnaire before and after intervention with the chewing gum (Table 2) and was significantly reduced after 2 weeks of intervention ( $p=0.05$ and $p=0.01$, respectively). Furthermore, an increase was reported in the subjective feeling of total amount of saliva in the mouth ( $p=0.007)$. In a total of 19 patients, $95 \%$ reported a subjective increase in saliva flow after intervention with the chewing gum. Eating difficulties in terms of swallowing issues were not found to differ significantly, whereas patients reported less trouble enjoying their meals after Visit $2(p=0.004)$.

\section{Salivary Output}

Mean distribution and individual data of saliva output for patients at Visit 1 are illustrated in Figure 1. At Visit 1, the mean unstimulated saliva output and stimulated saliva output were 0.79 and

TABLE 1 | Patient and tumor characteristics for all eligible patients.

\begin{tabular}{|c|c|c|c|c|c|c|}
\hline & Total $^{a}$ & Non-participants ${ }^{b}$ & Participants $^{c}$ & $p$ & Study groupd & $p^{\mathrm{e}}$ \\
\hline & $n=62$ & $n=31$ & $n=31$ & & $n=20$ & \\
\hline Men & $42(68 \%)$ & $18(58 \%)$ & $24(77 \%)$ & NS & $15(75 \%)$ & NS \\
\hline Age (median) (range, years) & $63(39-78)$ & $64(39-78)$ & $62(46-73)$ & NS & $62(46-73)$ & NS \\
\hline Smoking after RT & $10(16 \%)$ & $9(29 \%)$ & $1(3 \%)$ & 0.01 & 0 & NS \\
\hline \multicolumn{7}{|l|}{ Follow-up after RT } \\
\hline 2-5 months & $34(55 \%)$ & $18(58 \%)$ & $16(52 \%)$ & NS & $10(50 \%)$ & NS \\
\hline 6-9 months & $13(21 \%)$ & 7 (23\%) & $6(19 \%)$ & & 5 (25\%) & \\
\hline 10-12 months & $14(23 \%)$ & $7(23 \%)$ & $7(23 \%)$ & & $5(25 \%)$ & \\
\hline >12 months & $1(2 \%)$ & & $1(3 \%)$ & & & \\
\hline \multicolumn{7}{|l|}{ Site } \\
\hline Pharynx & $48(77 \%)$ & 25 (81\%) & $23(79 \%)$ & NS & $15(75 \%)$ & NS \\
\hline Oral cavity & $10(16 \%)$ & $5(16 \%)$ & $5(16 \%)$ & & $3(15 \%)$ & \\
\hline Saliva gland & $1(2 \%)$ & & $1(3 \%)$ & & - & \\
\hline Unknown primary tumor & $3(5 \%)$ & $1(3 \%)$ & $2(7 \%)$ & & $2(10 \%)$ & \\
\hline Clinical stage III-IV & $51(82 \%)$ & 27 (87\%) & $24(77 \%)$ & NS & $16(80 \%)$ & NS \\
\hline Concomitant chemotherapy & 32 (52\%) & $13(31 \%)$ & $19(61 \%)$ & NS & $10(50 \%)$ & 0.05 \\
\hline \multicolumn{7}{|l|}{ Distance to hospital } \\
\hline$>50 \mathrm{~km}$ & 24 (39\%) & 12 (36\%) & $12(41 \%)$ & NS & $10(50 \%)$ & NS \\
\hline$<50 \mathrm{~km}$ & $38(61 \%)$ & $20(65 \%)$ & 18 (58\%) & & $10(50 \%)$ & \\
\hline
\end{tabular}

aTotal number of consecutive and eligible patients asked to participated in the study.

${ }^{b}$ Number of patients declining to participate including screening failure.

"Number of patients consenting to participate including patients not completing Visit 2.

${ }^{d}$ Number of patients with repeating measurements who complete the study.

ep-Value comparing the patients completing the study with the patients lost to follow up.

$R T$, radiotherapy; $N S$, no significant $p$-value. 
TABLE 2 | Responses to the abbreviated EORTC H\&N35 questionnaire from participants completing the study ( $n=20)$.

\begin{tabular}{|c|c|c|c|c|c|c|c|c|c|c|}
\hline & & \multicolumn{4}{|c|}{ Visit 1} & \multicolumn{4}{|c|}{ Visit 2} & \multirow[t]{2}{*}{$p^{\mathrm{a}}$} \\
\hline \multicolumn{2}{|c|}{ In the past week have you had... } & None & A little & Quite a bit & A lot & None & A little & Quite a bit & A lot & \\
\hline \multicolumn{11}{|c|}{ Oral cavity } \\
\hline Q1 & Pain in your mouth? & $13(65 \%)$ & $4(20 \%)$ & $2(10 \%)$ & $1(5 \%)$ & $17(85 \%)$ & $2(10 \%)$ & $1(5 \%)$ & & 0.05 \\
\hline Q2 & Pain in your jaw? & $14(70 \%)$ & $4(20 \%)$ & $1(5 \%)$ & $1(5 \%)$ & $18(90 \%)$ & $1(5 \%)$ & $1(5 \%)$ & & 0.01 \\
\hline Q4 & A dry mouth? & $1(5 \%)$ & $1(5 \%)$ & $8(40 \%)$ & $10(50 \%)$ & 7 (35\%) & $7(35 \%)$ & $6(30 \%)$ & & NS \\
\hline Q5 & Sticky saliva? & $4(20 \%)$ & $8(40 \%)$ & $5(25 \%)$ & $3(15 \%)$ & $5(25 \%)$ & 7 (35\%) & 7 (35\%) & $1(5 \%)$ & NS \\
\hline Q6 & Less saliva? & $7(35 \%)$ & $2(10 \%)$ & $5(25 \%)$ & $6(30 \%)$ & $13(65 \%)$ & $3(15 \%)$ & $3(15 \%)$ & $1(5 \%)$ & 0.007 \\
\hline \multicolumn{11}{|c|}{ Eating difficulties } \\
\hline Q7 & Problems swallowing liquids? & $13(65 \%)$ & $6(30 \%)$ & $1(5 \%)$ & & $15(75 \%)$ & $4(20 \%)$ & $1(5 \%)$ & & NS \\
\hline $\mathrm{QB}^{\mathrm{x}}$ & Problems swallowing solid food? & $7(35 \%)$ & $5(25 \%)$ & $4(20 \%)$ & $3(15 \%)$ & $6(30 \%)$ & $8(40 \%)$ & $5(25 \%)$ & $1(5 \%)$ & NS \\
\hline Q11 & Decreased sense of taste? & $8(40 \%)$ & $4(20 \%)$ & $3(15 \%)$ & $5(25 \%)$ & $12(60 \%)$ & $4(20 \%)$ & $3(15 \%)$ & $1(5 \%)$ & 0.01 \\
\hline Q13 & Trouble enjoying your meals? & $5(25 \%)$ & $3(15 \%)$ & 7 (35\%) & $5(25 \%)$ & $6(30 \%)$ & $9(45 \%)$ & $4(20 \%)$ & $1(5 \%)$ & 0.004 \\
\hline Q14 & Trouble eating with other people? & $12(60 \%)$ & $4(20 \%)$ & $3(15 \%)$ & $1(5 \%)$ & $10(50 \%)$ & $6(30 \%)$ & $4(20 \%)$ & & NS \\
\hline Q19 & Increased saliva flow after using the chewing gum? & - & - & - & - & $\begin{array}{c}\text { "Yes" } \\
19 \text { (95\%) }\end{array}$ & $\begin{array}{c}\text { "No" } \\
1(5 \%)\end{array}$ & & & NS \\
\hline
\end{tabular}

Q6 and Q19 are added questions.

The $p$-value was found by using a paired T-test.

$Q$ is the number of the question in the questionnaire.

${ }^{x}$ Q8: one response is missing from questionnaire 1.

$N S$ is no significant $p$-value.

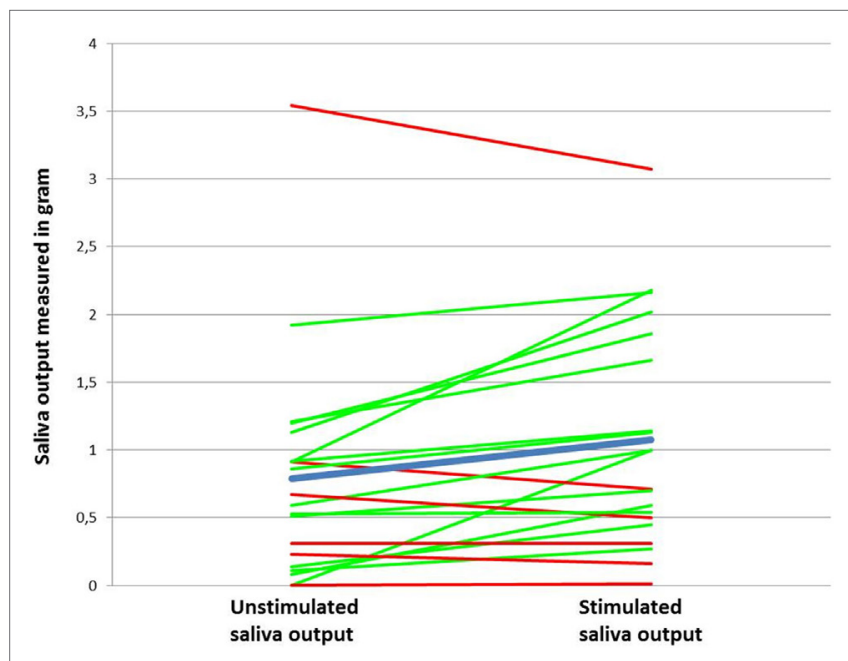

FIGURE 1 | Increase and decrease in saliva output after stimulation with the chewing gum $(\boldsymbol{n}=\mathbf{2 0})$ from Visit $\mathbf{1}$. The green line represents an increase in saliva output after stimulation. The red line represents a decrease or no change. The blue line represents the mean value.

$1.07 \mathrm{~g}$ versus 0.62 and $0.82 \mathrm{~g}$ at Visit 2 after intervention with the chewing gum (Figure 2). The increase in saliva output was found to be significant for both Visit 1 and 2 when tested by the twosided $t$-test ( $p=0.008$ and $p=0.05$, respectively). No significant difference in stimulated output between Visits 1 and 2 was found $(p=0.2)$. When testing mean saliva flow rate in grams per minute $(p=0.05$ and $p=0.04)$ and saliva volume $(p=0.02$ and $p=0.05)$, the increase in saliva was also found to be significant.

The mean flow rate for unstimulated saliva was $0.09 \mathrm{~mL} / \mathrm{min}$ after using chewing gum for 2 weeks. The definition for very low unstimulated whole saliva flow rate is $<0.1 \mathrm{~mL} / \mathrm{min}$ and corresponds with hyposalivation (6). Looking at all measurements of

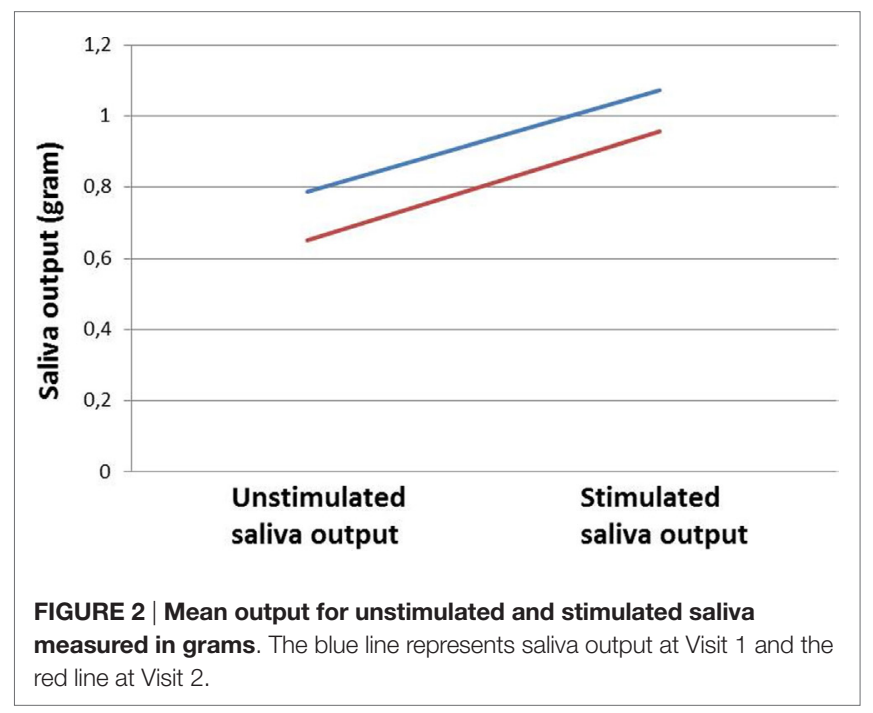

the unstimulated flow rate (milliliters per minute), nine patients had a very low flow rate below $0.1 \mathrm{~mL} / \mathrm{min}$, and seven patients had a low flow rate between 0.1 and $0.2 \mathrm{~mL} / \mathrm{min}$.

Saliva output for patients prescribed with xerostomia-induced medication $(n=5)$ did not seem to affect the results of chewing gum intervention, with mean unstimulated and stimulated saliva output being 1.25 and $1.52 \mathrm{~g}$ at Visit 1 .

Comparing the mean saliva output from the study group at Visit 1 and Visit 2 with the mean saliva output from the control group, an increase in saliva production was not seen in the control group. For the control group, both mean unstimulated and stimulated saliva output were much higher than the results presented in the study group (4.01 and $3.96 \mathrm{~g}$, respectively). No significant difference was found between mean unstimulated and stimulated saliva output for the control group. 


\section{DISCUSSION}

In this study, HNC patients treated with curative intended RT and concomitant chemotherapy had at least $0.2 \mathrm{~g}$ increase in saliva output after intervention with the tasteless and sugar-free chewing gum. For both Visit 1 and Visit 2, saliva output increased when stimulated by the chewing gum ( $p=0.008$ and $p=0.05$, respectively). This corresponded well with the patients reporting a positive subjective change regarding their xerostomia complaint. No special parotid sparing techniques were used for the patients included in the study. However, dose constraints to the parotid glands $\left[D_{\text {mean }} \leq 20 \mathrm{~Gy}\right.$ (contralateral) or $D_{\text {mean }} \leq 26 \mathrm{~Gy}$ (both)], submandibular glands $\left(D_{\text {mean }} \leq 35 \mathrm{~Gy}\right)$, and oral cavity ( $D_{\text {mean }} \leq 30 \mathrm{~Gy}$ ) were intended to be met in every single case.

With xerostomia being both an early and late side effect to primary curative intended RT for oral and oropharyngeal carcinomas, it is common that many patients will experience a permanent subjective feeling of dry mouth. Xerostomia is not a life-threatening complication; however, decreased saliva flow and changes in saliva composition are straining. Saliva often become sticky and unmanageable, which compromises oral well-being and results in eating difficulties, increased risk of caries, and impaired speaking abilities. Living with xerostomia may inflict emotional strain such as worry, tension, and depression and limit social activities $(10,12,15)$.

Various salivary substitutes and stimulants are on the market to relieve discomfort associated with xerostomia. Substitutes, such as sprays, gels, mouthwashes, and special toothpaste, are developed to keep soft tissue moist and increase the viscosity of liquids (16). Substitutes are limited by their short duration of effect, often unpleasant taste, and high cost but can be useful during the night (17). The saliva stimulants include sugar-free chewing gum, ascorbic acid (vitamin $\mathrm{C}$ tablets), malic acid, and pilocarpine (18). There is low level of evidence concerning the efficiency of the various products available.

For HNC patients, it remains a challenge to find suitable products to increase salivary flow. After ending RT, the oral cavity is highly sensitive due to decreased salivary flow rate and alterations to the sense of taste. Strong flavors, such as peppermint or lemon, are not favored in the early phase of recovery (19). Sugar may contribute to or worsen xerostomia and increases the risk of carries. The chewing muscles can be restrained due to radiation sequelae, and some patients are confined to processing small food items.

The chewing gum designed for this study tries to accommodate the need of the HNC patient. The lack of taste serves the purpose of stimulating the salivary flow by physical and mechanical stimulation alone, whereas the soft texture and small size makes it easy to chew regardless of rigid muscles or oral sensitivity. This is in agreement with others suggesting that saliva stimulants in the form of a sugar-free chewing gum can aid to promote salivation when residual salivary glandular function remains (20). The gum increases salivary flow by stimulating the taste receptors and through physical stimulation of the salivary gland $(12,18,21)$.

When comparing mean unstimulated and stimulated saliva output for both Visit 1 and Visit 2, a relative similar increase in salivary output was seen at both visits (Figure 2). The study was not able to find an absolute increase in whole unstimulated saliva output after the second visit, indicating that 2 weeks of regular use of chewing gum did not increase unstimulated saliva output. The decline may be explained by the short period of time allotted for the patient to adapt to the chewing gum, failure to use it on a regular basis, or progressing xerostomia after RT. Furthermore, the study from Flink et al. argues that the circadian rhythm and fasting may also have a negative impact on the saliva secretion in patients with hyposalivation (6). In this study, measurement of saliva flow took place throughout the day time with no regard to the circadian rhythm of saliva secretion. Due to scheduling difficulties, it was not possible to conduct Visit 2 at the exact same time slot or location for all study patients.

Despite including 31 eligible HNC patients, only 20 patients completed the study. After completing treatment for HNC, the patients are only frequenting the department for scheduled checkups with a span of 3-6 months. Participating in this study required an extra visit to the hospital for saliva collection and evaluation of the chewing gum alone. Some of the patients lost to follow-up did not allow time for an extra visit due to long travel distances or coincide with work.

The study did not look into changes in the saliva composition, and further studies should include testing the variations of saliva composition. The chewing gum used in this study did not include any lubricating or fluoride additives. Further testing ought to include chewing gum with emollient additives in order to see whether it is possible to make changes in the saliva composition and further improve the oral well-being.

\section{CONCLUSION}

Using chewing gum as a saliva stimulant, the study was able to stimulate and increase mean salivary output for 14 out of 20 consecutive HNC patients. The majority of the patients reported a self-rated improvement regarding xerostomia complaints and improved oral well-being after being subjected to the study chewing gum.

\section{AUTHOR CONTRIBUTIONS}

All the three authors have been actively involved in the design of the study, analyzing of the data, and writing the manuscript. Dr. JK and Dr. JE have been actively involved in the collection of data.

\section{ACKNOWLEDGMENTS}

The authors of this article would like to thank Fertin Pharma A/S who has developed and produced the chewing gum and has been an active partner in the project. Fertin Pharma A/S is part of the Danish Bagger-Sørensen group and has filed a patent for the product and chewing gum formula used in this study.

\section{FUNDING}

The study was funded by the Department of Oncology, OUH, and provided facilities during the study. No grant was provided for the study. Fertin Pharma produced the chewing gum free of charge. 


\section{REFERENCES}

1. Mira JG, Fullerton GD, Wescott WB. Correlation between initial salivary flow rate and radiation dose in the production of xerostomia. Acta Radiol Oncol (1982) 21(3):151-4. doi:10.3109/02841868209133999

2. Jellema AP, Doornaert P, Slotman BJ, Leemans CR, Langendijk JA. Does radiation dose to the salivary glands and oral cavity predict patient-rated xerostomia and sticky saliva in head and neck cancer patients treated with curative radiotherapy? Radiother Oncol (2005) 77(2):164-71. doi:10.1016/j. radonc.2005.10.002

3. Porter SR, Fedele S, Habbab KM. Xerostomia in head and neck malignancy. Oral Oncol (2010) 46(6):460-3. doi:10.1016/j.oraloncology.2010.03.008

4. Nieuw Amerongen AV, Veerman EC. Current therapies for xerostomia and salivary gland hypofunction associated with cancer therapies. Support Care Cancer (2003) 11(4):226-31. doi:10.1007/s00520-002-0409-5

5. Wolff M, Kleinberg I. Oral mucosal wetness in hypo- and normosalivators. Arch Oral Biol (1998) 43(6):455-62. doi:10.1016/S0003-9969(98)00022-3

6. Flink H, Tegelberg A, Lagerlof F. Influence of the time of measurement of unstimulated human whole saliva on the diagnosis of hyposalivation. Arch Oral Biol (2005) 50(6):553-9. doi:10.1016/j.archoralbio.2004.10.015

7. Navazesh M, Kumar SK. Measuring salivary flow: challenges and opportunities. J Am Dent Assoc (2008) 139(Suppl):35s-40s. doi:10.14219/jada. archive.2008.0353

8. Saleh J, Figueiredo MA, Cherubini K, Salum FG. Salivary hypofunction: an update on aetiology, diagnosis and therapeutics. Arch Oral Biol (2014) 60(2):242-55. doi:10.1016/j.archoralbio.2014.10.004

9. Mortensen HR, Overgaard J, Specht L, Overgaard M, Johansen J, Evensen JF, et al. Prevalence and peak incidence of acute and late normal tissue morbidity in the DAHANCA 6\&7 randomised trial with accelerated radiotherapy for head and neck cancer. Radiother Oncol (2012) 103(1):69-75. doi:10.1016/j. radonc.2012.01.002

10. Jensen AB, Hansen O, Jorgensen K, Bastholt L. Influence of late side-effects upon daily life after radiotherapy for laryngeal and pharyngeal cancer. Acta Oncol (1994) 33(5):487-91. doi:10.3109/02841869409083923

11. Deasy JO, Moiseenko V, Marks L, Chao KS, Nam J, Eisbruch A. Radiotherapy dose-volume effects on salivary gland function. Int J Radiat Oncol Biol Phys (2010) 76(3 Suppl):S58-63. doi:10.1016/j.ijrobp.2009.06.090

12. Dirix P, Nuyts S, Vander Poorten V, Delaere P, Van den Bogaert W. The influence of xerostomia after radiotherapy on quality of life: results of a questionnaire in head and neck cancer. Support Care Cancer (2008) 16(2):171-9. doi:10.1007/s00520-007-0300-5
13. Bjordal K, Hammerlid E, Ahlner-Elmqvist M, de Graeff A, Boysen M, Evensen JF, et al. Quality of life in head and neck cancer patients: validation of the European Organization for Research and Treatment of Cancer Quality of Life Questionnaire-H\&N35. J Clin Oncol (1999) 17(3):1008-19.

14. Bjordal K, de Graeff A, Fayers PM, Hammerlid E, van Pottelsberghe C, Curran D, et al. A 12 country field study of the EORTC QLQ-C30 (version 3.0) and the head and neck cancer specific module (EORTC QLQ-H\&N35) in head and neck patients. EORTC Quality of Life Group. Eur J Cancer (2000) 36(14):1796-807. doi:10.1016/S0959-8049(00)00186-6

15. Jensen K, Jensen AB, Grau C. A cross sectional quality of life study of 116 recurrence free head and neck cancer patients. The first use of EORTC H\&N35 in Danish. Acta Oncol (2006) 45(1):28-37. doi:10.1080/02841860500417536

16. Vissink A, Mitchell JB, Baum BJ, Limesand KH, Jensen SB, Fox PC, et al. Clinical management of salivary gland hypofunction and xerostomia in headand-neck cancer patients: successes and barriers. Int J Radiat Oncol Biol Phys (2010) 78(4):983-91. doi:10.1016/j.ijrobp.2010.06.052

17. Chambers MS, Rosenthal DI, Weber RS. Radiation-induced xerostomia. Head Neck (2007) 29(1):58-63. doi:10.1002/hed.20456

18. Visvanathan V,NixP.Managing thepatient presenting with xerostomia:a review. Int J Clin Pract (2010) 64(3):404-7. doi:10.1111/j.1742-1241.2009.02132.x

19. Tribius S, Raguse M, Voigt C, Munscher A, Grobe A, Petersen C, et al. Residual deficits in quality of life one year after intensity-modulated radiotherapy for patients with locally advanced head and neck cancer: results of a prospective study. Strahlenther Onkol(2015) 191(6):501-10. doi:10.1007/s00066-015-0824-4

20. Dost F, Farah CS. Stimulating the discussion on saliva substitutes: a clinical perspective. Aust Dent J (2013) 58(1):11-7. doi:10.1111/adj.12023

21. Plemons JM, Al-Hashimi I, Marek CL. Managing xerostomia and salivary gland hypofunction: executive summary of a report from the American Dental Association Council on Scientific Affairs. J Am Dent Assoc (2014) 145(8):867-73. doi:10.14219/jada.2014.44

Conflict of Interest Statement: The authors declare that the research was conducted in the absence of any commercial or financial relationships that could be construed as a potential conflict of interest.

Copyright (c) 2016 Kaae, Stenfeldt and Eriksen. This is an open-access article distributed under the terms of the Creative Commons Attribution License (CC BY). The use, distribution or reproduction in other forums is permitted, provided the original author(s) or licensor are credited and that the original publication in this journal is cited, in accordance with accepted academic practice. No use, distribution or reproduction is permitted which does not comply with these terms. 